\title{
The universal covering homomorphism in o-minimal expansions of groups
}

\author{
Mário J. Edmundo* \\ CMAF Universidade de Lisboa \\ Av. Prof. Gama Pinto 2 \\ 1649-003 Lisboa, Portugal \\ edmundo@cii.fc.ul.pt
}

\author{
Pantelis E. Eleftheriou ${ }^{\dagger}$ \\ Department of Mathematics \\ University of Notre Dame \\ Notre Dame, IN 46556, USA \\ pelefthe@nd.edu
}

February 19, 2006

\begin{abstract}
Suppose that $G$ is a definably connected, definable group in an ominimal expansion of an ordered group. We show that the o-minimal universal covering homomorphism $\widetilde{p}: \widetilde{G} \longrightarrow G$ is a locally definable covering homomorphism and $\pi_{1}(G)$ is isomorphic to the o-minimal fundamental group $\pi(G)$ of $G$ defined using locally definable covering homomorphisms.
\end{abstract}

\footnotetext{
${ }^{*}$ With partial support from the FCT (Fundação para a Ciência e Tecnologia), program POCTI (Portugal/FEDER-EU).

$\dagger$ Partially supported by NSF grant DMS-02-45167 (Cholak). MSC: 03C64, 20E99. Keywords and phrases: O-minimal structures, universal covers, definable groups. Revised: June 1, 2007.
} 


\section{Introduction}

Let $\mathcal{R}$ be an o-minimal expansion of an ordered group $(R, 0,+,<)$. The structure $\mathcal{R}$ will be fixed throughout and will be assumed to be $\aleph_{1}$-saturated. By definable we will mean definable in $\mathcal{R}$ with parameters.

In the paper [3] the first author introduced a notion of o-minimal fundamental group and o-minimal universal covering homomorphism for definable groups (or more generally for locally definable groups) in arbitrary o-minimal structures which we now recall.

First recall that a group $(G, \cdot)$ is a locally definable group over $A$, with $A \subseteq R$ and $|A|<\aleph_{1}$, if there is a countable collection $\left\{Z_{i}: i \in I\right\}$ of definable subsets of $R^{n}$, all definable over $A$, such that: (i) $G=\cup\left\{Z_{i}: i \in I\right\}$; (ii) for every $i, j \in I$ there is $k \in I$ such that $Z_{i} \cup Z_{j} \subseteq Z_{k}$ and (iii) the restriction of the group multiplication to $Z_{i} \times Z_{j}$ is a definable map over $A$ into $R^{n}$.

Given two locally definable groups $H$ and $G$ over $A$, we say that $H$ is a locally definable subgroup of $G$ over $A$ if $H$ is a subgroup of $G$.

A homomorphism $\alpha: G \longrightarrow H$ between locally definable groups over $A$ is called a locally definable homomorphism over $A$ if for every definable subset $Z \subseteq G$ defined over $A$, the restriction $\alpha_{\mid Z}$ is a definable map over $A$.

In the terminology of [9], locally definable groups (respectively homomorphisms) are $\bigvee$-definable groups (respectively homomorphisms). Therefore, every locally definable group $G \subseteq R^{n}$ over $A$ is equipped with a unique topology $\tau$, called the $\tau$-topology, such that: (i) $(G, \tau)$ is a topological group; (ii) every generic element of $G$ has an open definable neighborhood $U \subseteq R^{n}$ such that $U \cap G$ is $\tau$-open and the topology which $U \cap G$ inherits from $\tau$ agrees with the topology it inherits from $R^{n}$; (iii) locally definable homomorphisms between locally definable groups are continuous with respect to the $\tau$ topologies. Note also that when $G$ is a definable group, then its $\tau$-topology coincides with the its t-topology from [10].

Definition 1.1 A locally definable homomorphism $p: H \longrightarrow G$ over $A$ between locally definable groups over $A$ is called a locally definable covering homomorphism if $p$ is surjective and there is a family $\left\{U_{l}: l \in L\right\}$ of $\tau$-open definable subsets of $G$ over $A$ such that $G=\cup\left\{U_{l}: l \in L\right\}$ and, for each $l \in L, p^{-1}\left(U_{l}\right)$ is a disjoint union of $\tau$-open definable subsets of $H$ over $A$, each of which is mapped homeomorphically by $p$ onto $U_{l}$.

We call $\left\{U_{l}: l \in L\right\}$ a $p$-admissible family of definable $\tau$-neighborhoods over $A$.

We denote by $\operatorname{Cov}(G)$ the category whose objects are locally definable covering homomorphisms $p: H \longrightarrow G$ (over some $A$ with $|A|<\aleph_{1}$ ) and 
whose morphisms are surjective locally definable homomorphisms $r: H \longrightarrow$ $K$ (over some $A$ with $|A|<\aleph_{1}$ ) such that $q \circ r=p$, where $q: K \longrightarrow G$ is a locally definable covering homomorphism (over some $A$ with $|A|<\aleph_{1}$ ). Let $p: H \longrightarrow G$ and $q: K \longrightarrow G$ be locally definable covering homomorphisms. If $r: H \longrightarrow K$ is a morphism in $\operatorname{Cov}(G)$, then by [3] Theorem 3.6, $r: H \longrightarrow$ $K$ is a locally definable covering homomorphism.

Definition 1.2 The category $\operatorname{Cov}(G)$ and its full subcategory $\operatorname{Cov}^{0}(G)$ with objects $h: H \longrightarrow G$ such that $H$ is a definably connected locally definable group, form inverse systems ([3] Corollary 3.7 and Lemma 3.8). The inverse limit $\widetilde{p}: \widetilde{G} \longrightarrow G$ of the inverse system $\operatorname{Cov}^{0}(G)$ is called the (o-minimal) universal covering homomorphism of $G$.

The kernel of the universal covering homomorphism $\widetilde{p}: \widetilde{G} \longrightarrow G$ of $G$ is called the (o-minimal) fundamental group of $G$ and is denoted by $\pi(G)$.

Inverse limits of inverse systems of groups always exist in the category of groups ([11] Proposition 1.1.1), but in general we do not know if the ominimal universal covering homomorphism $\widetilde{p}: \widetilde{G} \longrightarrow G$ is locally definable. The main result of this paper is that this is the case in o-minimal expansions of groups.

On the other hand, in the paper [5], the second author and S. Starchenko use definable $t$-continuous paths to define the o-minimal fundamental group $\pi_{1}(G)$ of a definably $t$-connected, definable group $G$ following the classical case in [7] and the case in o-minimal expansions of fields treated by Berarducci and Otero in [1]. We will adapt that definition to the category of locally definable groups. As in [5] we will run the definition in parallel with respect to the $\tau$-topology of a definably connected locally definable group $G$ and the usual topology on an arbitrary definable subset $X$ of $R^{n}$.

A $(\tau$-)path $\alpha:[0, p] \longrightarrow X(\alpha:[0, p] \longrightarrow G)$ is a $(\tau$-)continuous definable map. A $(\tau$-)path $\alpha:[0, p] \longrightarrow X(\alpha:[0, p] \longrightarrow G)$ is a $(\tau$-)loop if $\alpha(0)=$ $\alpha(p)$. A concatenation of two $(\tau-)$ paths $\gamma:[0, p] \longrightarrow X(\gamma:[0, p] \longrightarrow G)$ and $\delta:[0, q] \longrightarrow X(\delta:[0, q] \longrightarrow G)$ with $\gamma(p)=\delta(0)$ is a $(\tau$-)path $\gamma \cdot \delta:[0, p+q] \longrightarrow X(\gamma \cdot \delta:[0, p+q] \longrightarrow G)$ with:

$$
(\gamma \cdot \delta)(t)= \begin{cases}\gamma(t) & \text { if } t \in[0, p] \\ \delta(t-p) & \text { if } t \in[p, p+q] .\end{cases}
$$

Given two definable $\left(\tau\right.$-)continuous maps $f, g: Y \subseteq R^{m} \longrightarrow X(f, g: Y \subseteq$ $\left.R^{m} \longrightarrow G\right)$, we say that a definable $(\tau$-)continuous map $F(t, s): Y \times[0, q] \longrightarrow$ $X(F(t, s): Y \times[0, q] \longrightarrow G)$, is a $\left(\tau\right.$-)homotopy between $f$ and $g$ if $f=F_{0}$ and $g=F_{q}$, where $\forall s \in[0, q], F_{s}:=F(\cdot, s)$. In this situation we say that $f$ and $g$ are $(\tau-)$ homotopic, denoted $f \sim g\left(f \sim_{\tau} g\right)$. 
Definition 1.3 Two $(\tau-)$ paths $\gamma:[0, p] \longrightarrow X(\gamma:[0, p] \longrightarrow G), \delta:$ $[0, q] \longrightarrow X(\delta:[0, q] \longrightarrow G)$, with $\gamma(0)=\delta(0)$ and $\gamma(p)=\delta(q)$, are called $\left(\tau\right.$-)homotopic if there is some $t_{0} \in[0, \min \{p, q\}]$, and a $(\tau$-) homotopy $F(t, s):[0, \max \{p, q\}] \times[0, r] \longrightarrow X(F(t, s):[0, \max \{p, q\}] \times[0, r] \longrightarrow G)$, for some $r>0$ in $R$, between

$$
\begin{aligned}
& \left.\gamma_{\left[0, t_{0}\right]} \cdot \mathbf{c} \cdot \gamma_{\left[\left[t_{0}, p\right]\right.} \text { and } \delta \text { (if } p \leq q\right), \text { or } \\
& \delta_{\left[\left[0, t_{0}\right]\right.} \cdot \mathbf{d} \cdot \delta_{\mid\left[t_{0}, q\right]} \text { and } \gamma(\text { if } q \leq p) .
\end{aligned}
$$

where $\mathbf{c}(t)=\gamma\left(t_{0}\right)$ and $\mathbf{d}(t)=\delta\left(t_{0}\right)$ are the constant $(\tau$-)paths with domain $[0,|p-q|]$.

If $\mathbb{L}(G)$ denotes the set of all $\tau$-loops that start and end at the identity element $e_{G}$ of $G$, the restriction of $\sim_{\tau}$ to $\mathbb{L}(G) \times \mathbb{L}(G)$ is an equivalence relation on $\mathbb{L}(G)$. We define

$$
\pi_{1}(G):=\mathbb{L}(G) / \sim_{\tau}
$$

and $[\gamma]:=$ the class of $\gamma \in \mathbb{L}(G)$. Note that $\pi_{1}(G)$ is indeed a group with group operation given by $[\gamma][\delta]=[\gamma \cdot \delta]$.

In a similar way we define the o-minimal fundamental group $\pi_{1}(X)$ of a definable set $X \subseteq R^{n}$.

Given the above two possible definitions of o-minimal fundamental groups it is natural to try to find out if they coincide. Our main result shows that this is the case:

Theorem 1.4 Let $\mathcal{R}$ be an o-minimal expansion of a group and $G$ a definably t-connected definable group. Then the o-minimal universal covering homomorphism $\widetilde{p}: \widetilde{G} \longrightarrow G$ is a locally definable covering homomorphism and $\pi_{1}(G)$ is isomorphic to $\pi(G)$.

Theorem 1.4 will actually be proved for definably $\tau$-connected locally definable groups. See Theorem 3.11 below. As a consequence of our work we obtain the following corollary which is proved at the end of the paper.

Corollary 1.5 Let $\mathcal{R}$ be an o-minimal expansion of a group and $G$ a definably $t$-connected definable group. Then $\pi_{1}(G)$ is a finitely generated abelian group. Moreover, if $G$ is abelian, then there is $l \in \mathbb{N}$ such that $\pi_{1}(G) \simeq \mathbb{Z}^{l}$ and, for each $k \in \mathbb{N}$, the subgroup $G[k]$ of $k$-torsion points of $G$ is given by $G[k] \simeq(\mathbb{Z} / k \mathbb{Z})^{l}$.

When $G$ is a definably compact, abelian definable group, we conjecture that $l$ above is the dimension of $G$. This is known to be the case when $\mathcal{R}$ is linear ([5]) or $\mathcal{R}$ is an o-minimal expansion of a real closed field ([4]). So the conjecture is open for $\mathcal{R}$ eventually linear but not linear. 


\section{Preliminary results}

This section contains all the lemmas that come from other references and are used later in the paper. Thus we generalize the theory of [3] and [4] Section 2 to the category of locally definable covering maps of locally definable groups in $\mathcal{R}$. Since the arguments are similar we will omit the details.

Definition 2.1 A set $Z$ is a locally definable set over $A$, where $A \subseteq R$ and $|A|<\aleph_{1}$, if there is a countable collection $\left\{Z_{i}: i \in I\right\}$ of definable subsets of $R^{n}$, all definable over $A$, such that: (i) $Z=\cup\left\{Z_{i}: i \in I\right\}$; (ii) for every $i, j \in I$ there is $k \in I$ such that $Z_{i} \cup Z_{j} \subseteq Z_{k}$.

Given two locally definable sets $X$ and $Z$ over $A$, we say that $X$ is a locally definable subset of $Z$ over $A$ if $X$ is a subset of $Z$.

A map $\alpha: Z \longrightarrow X$ between locally definable sets over $A$ is called a locally definable map over $A$ if for every definable subset $V \subseteq Z$ defined over $A$, the restriction $\alpha_{\mid V}$ is a definable map over $A$.

By saturation, the set $Z$ does not depend on the choice of the collection $\left\{Z_{i}: i \in I\right\}$. Furthermore, if $\alpha: Z \longrightarrow X$ is a locally definable map over $A$ between locally definable sets over $A$ and $Y$ is a locally definable subset of $X$ over $A$, then the following hold:

(1) $\alpha(Z)$ is a locally definable subset of $X$ over $A$ and $\alpha^{-1}(Y)$ is a locally definable subset of $Z$ over $A$.

(2) If $Y$ is such that $V \cap Y$ is definable for every definable subset $V$ of $X$, then $W \cap \alpha^{-1}(Y)$ is definable for every definable subset $W$ of $Z$. (Since $\left.\left.W \cap \alpha^{-1}(Y)=\alpha_{\mid W}^{-1}(\alpha(W) \cap Y)\right)\right)$.

Definition 2.2 Let $G$ be a locally definable group over $A$ and $W$ a locally definable set over $A$. A locally definable map $w: W \longrightarrow G$ over $A$ is called a locally definable covering map if $w$ is surjective and there is a family $\left\{U_{l}: l \in\right.$ $L\}$ of $\tau$-open definable subsets of $G$ over $A$ such that $G=\cup\left\{U_{l}: l \in L\right\}$ and, for each $l \in L$, the locally definable subset $w^{-1}\left(U_{l}\right)$ of $W$ over $A$ is a disjoint union of definable subsets of $W$ over $A$, each of which is mapped bijectively by $w$ onto $U_{l}$.

We call $\left\{U_{l}: l \in L\right\}$ a $w$-admissible family of definable $\tau$-neighborhoods over $A$.

Given a locally definable covering map $w: W \longrightarrow G$ over $A$ there is a topology on $W$, which we call the $w$-topology, generated by the definable sets of the form $w^{-1}(U) \cap V$, where $U$ is a $\tau$-open definable subset of $G$ and $V$ is one of the definable subsets of the disjoint union $w^{-1}\left(U_{l}\right)$ for some $U_{l}$ in the $w$-admissible family of definable $\tau$-neighborhoods. 
Clearly, with respect to the $w$-topology on $W$ (and the $\tau$-topology on $G), w: W \longrightarrow G$ is continuous. Furthermore, $w: W \longrightarrow G$ is an open surjection. In fact, let $V$ be a $w$-open definable subset of $W$ over $A$ and, for each $l \in L$, let $\left\{U_{s}^{l}: s \in S_{l}\right\}$ be the collection of $w$-open disjoint definable subsets of $W$ over $A$ such that $w^{-1}\left(U_{l}\right)=\cup\left\{U_{s}^{l}: s \in S_{l}\right\}$ and $w_{\mid U_{s}^{l}}: U_{s}^{l} \longrightarrow U_{l}$ is a definable homeomorphism over $A$ for every $s \in S_{l}$. Since $|A|<\aleph_{1}$, by saturation, there is $\left\{W_{1}, \ldots, W_{m}\right\} \subseteq\left\{U_{s}^{l}: l \in L, s \in S_{l}\right\}$ such that $V \subseteq \cup\left\{W_{i}: i=1, \ldots, m\right\}$. But then $V=\cup\left\{V \cap W_{i}: i=1, \ldots, m\right\}$ and $w(V)=\cup\left\{w\left(V \cap W_{i}\right): i=1, \ldots, m\right\}$ is $\tau$-open.

Lemma 2.3 Let $w: W \longrightarrow G$ be a locally definable covering map and suppose that $W$ is also a locally definable group. Then on $W$ the $w$-topology coincides with the $\tau$-topology.

Proof. Let $a \in W$ be a generic point and $U$ a definable $w$-open neighborhood of $a$ in $W$. We may assume that $w_{\mid U}: U \longrightarrow w(U)$ is a definable homeomorphism. Since $w(a)$ is also generic, there exists a definable subset $V \subseteq w(U)$ containing $w(a)$ such that $V$ is both $\tau$-open in $G$ and open in $G$ with the induced topology on $G$ from $R^{n}$. Thus $w^{-1}(V)$ is also both a $w$ neighborhood of $a$ in $W$ and in $W$ with the induced topology on $W$ from $R^{n}$. Hence, $w^{-1}(V)$ is a $\tau$-neighborhood of $a$ in $W$. By uniqueness of $\tau$-topology, this implies that the $w$-topology and the $\tau$-topology on $W$ agree.

Let $w: W \longrightarrow G$ be a locally definable covering map (over some $A$ with $\left.|A|<\aleph_{1}\right)$. Let $X$ be a definable subset of $W$ equipped with the induced $w$-topology from $W$. We will now introduce certain notions in parallel for $X$ and $W$.

A $w$-path $\alpha:[0, p] \longrightarrow X(\alpha:[0, p] \longrightarrow W)$ is a $w$-continuous definable map. A $w$-path $\alpha:[0, p] \longrightarrow X(\alpha:[0, p] \longrightarrow W)$ is a $w$-loop if $\alpha(0)=\alpha(p)$. A concatenation of two $w$-paths $\gamma:[0, p] \longrightarrow X(\gamma:[0, p] \longrightarrow W)$ and $\delta:[0, q] \longrightarrow X(\delta:[0, q] \longrightarrow W)$ with $\gamma(p)=\delta(0)$ is a $w$-path $\gamma \cdot \delta:$ $[0, p+q] \longrightarrow X(\gamma \cdot \delta:[0, p+q] \longrightarrow W)$ with:

$$
(\gamma \cdot \delta)(t)= \begin{cases}\gamma(t) & \text { if } t \in[0, p] \\ \delta(t-p) & \text { if } t \in[p, p+q]\end{cases}
$$

Given two definable $w$-continuous maps $f, g: Y \subseteq R^{m} \longrightarrow X(f, g: Y \subseteq$ $\left.R^{m} \longrightarrow W\right)$, we say that a definable $w$-continuous map $F(t, s): Y \times[0, q] \longrightarrow$ $X(F(t, s): Y \times[0, q] \longrightarrow W)$ is a $w$-homotopy between $f$ and $g$ if $f=F_{0}$ and $g=F_{q}$, where $\forall s \in[0, q], F_{s}:=F(\cdot, s)$. In this situation we say that $f$ and $g$ are $w$-homotopic, denoted $f \sim_{w} g$. 
Definition 2.4 Two $w$-paths $\gamma:[0, p] \longrightarrow X(\gamma:[0, p] \longrightarrow W), \delta:[0, q] \longrightarrow$ $X(\delta:[0, q] \longrightarrow W)$, with $\gamma(0)=\delta(0)$ and $\gamma(p)=\delta(q)$, are called $w$ homotopic if there is some $t_{0} \in[0, \min \{p, q\}]$, and a $w$-homotopy $F(t, s)$ : $[0, \max \{p, q\}] \times[0, r] \longrightarrow X(F(t, s):[0, \max \{p, q\}] \times[0, r] \longrightarrow W)$, for some $r>0$ in $R$, between

$$
\begin{aligned}
& \left.\gamma_{\left[\left[0, t_{0}\right]\right.} \cdot \mathbf{c} \cdot \gamma_{\mid\left[t_{0}, p\right]} \text { and } \delta \text { (if } p \leq q\right) \text {, or } \\
& \delta_{\left[\left[0, t_{0}\right]\right.} \cdot \mathbf{d} \cdot \delta_{\left[\left[t_{0}, q\right]\right.} \text { and } \gamma(\text { if } q \leq p) .
\end{aligned}
$$

where $\mathbf{c}(t)=\gamma\left(t_{0}\right)$ and $\mathbf{d}(t)=\delta\left(t_{0}\right)$ are the constant $w$-paths with domain $[0,|p-q|]$.

If $\mathbb{L}(W)$ denotes the set of all $w$-loops that start and end at a fixed element $e_{W}$ of $W$ such that $w\left(e_{W}\right)=e_{G}$, the restriction of $\sim_{w}$ to $\mathbb{L}(W) \times \mathbb{L}(W)$ is an equivalence relation on $\mathbb{L}(W)$. We define

$$
\pi_{1}(W):=\mathbb{L}(W) / \sim_{w}
$$

and $[\gamma]:=$ the class of $\gamma \in \mathbb{L}(W)$. Note that $\pi_{1}(W)$ is indeed a group with group operation given by $[\gamma][\delta]=[\gamma \cdot \delta]$. Also this group depends on the $w$-topology on $W$.

In a similar way we define the o-minimal fundamental group $\pi_{1}(X)$ of a definable subset $X \subseteq W$ with respect to the induced $w$-topology.

Clearly, any two constant $w$-loops at the same point $c \in W$ are $w$ homotopic. We will thus write $\epsilon_{c}$ for the constant $w$-loop at $c$ without specifying its domain.

In view of Lemma 2.3, we obtain the above notions with $w$ replaced by $\tau$ for definable subsets of a locally definable group equipped with the induced $\tau$-topology.

Lemma 2.5 Let $w: W \longrightarrow G$ and $v: V \longrightarrow H$ be locally definable covering maps. Then $(w, v): W \times V \longrightarrow G \times H$ is a locally definable covering map and $\theta: \pi_{1}(W) \times \pi_{1}(V) \longrightarrow \pi_{1}(W \times V):([\gamma],[\delta]) \mapsto[(\gamma, \delta)]$ is a group isomorphism.

Proof. The inverse of $\theta$ is $\pi_{1}(W \times V) \longrightarrow \pi_{1}(W) \times \pi_{1}(V):[\rho] \mapsto$ $\left(\left[q_{1} \circ \rho\right],\left[q_{2} \circ \rho\right]\right)$ where $q_{1}$ and $q_{2}$ are the projections from $W \times V$ onto $W$ and $V$, respectively.

Let $w: W \longrightarrow G$ be a locally definable covering map (over some $A$ with $|A|<\aleph_{1}$ ). Let $Z$ be a definable set and let $f: Z \longrightarrow G$ be a definable 
continuous map (with respect to the $\tau$-topology on $G$ ). A lifting of $f$ is a continuous definable map $\tilde{f}: Z \longrightarrow W$ (with respect to the $w$-topology on $W$ ) such that $p \circ \tilde{f}=f$.

Lemma 2.6 Let $w: W \longrightarrow G$ be a locally definable covering map, $Z$ a definably connected definable set and $f: Z \longrightarrow G$ a definable continuous map. If $\widetilde{f}_{1}, \widetilde{f}_{2}: Z \longrightarrow W$ are two liftings of $f$, then $\widetilde{f}_{1}=\widetilde{f}_{2}$ provided there is $a z \in Z$ such that $\tilde{f}_{1}(z)=\widetilde{f}_{2}(z)$.

Proof. As in the proof of [3] Lemma 3.2, both sets $\left\{w \in Z: \widetilde{f}_{1}(w)=\right.$ $\left.\tilde{f}_{2}(w)\right\}$ and $\left\{w \in Z: \widetilde{f}_{1}(w) \neq \widetilde{f}_{2}(w)\right\}$ are definable and open, the first one is nonempty.

Lemma 2.7 Suppose that $w: W \longrightarrow G$ is a locally definable covering map. Then the following hold.

(1) Let $\gamma$ be a $\tau$-path in $G$ and $y \in W$. If $w(y)=\gamma(0)$, then there is a unique w-path $\widetilde{\gamma}$ in $W$, lifting $\gamma$, such that $\widetilde{\gamma}(0)=y$.

(2) Suppose that $F$ is a $\tau$-homotopy between the $\tau$-paths $\gamma$ and $\sigma$ in $G$. Let $\widetilde{\gamma}$ be a w-path in $W$ lifting $\gamma$. Then there is a unique definable lifting $\widetilde{F}$ of $F$, which is a w-homotopy between $\widetilde{\gamma}$ and $\widetilde{\sigma}$, where $\widetilde{\sigma}$ is a w-path in $W$ lifting $\sigma$.

Proof. In our category, the path and the homotopy liftings can be proved as in [4] by observing that, by saturation, a definable subset of $G$ is covered by finitely many open definable subsets of $G$.

Notation: Referring to Lemma 2.7, if $\gamma:[0, q] \longrightarrow G$ is a $\tau$-path in $G$ and $y \in W$, we denote by $y * \gamma$ the final point $\widetilde{\gamma}(q)$ of the lifting $\widetilde{\gamma}$ of $\gamma$ with initial point $\widetilde{\gamma}(0)=y$.

The following consequence of Lemma 2.7 is proved in exactly the same way as its definable analogue in [4] Corollary 2.9. Below, for $w: W \longrightarrow G$ a locally definable covering map, we say that $W$ is definably $w$-connected if there is no proper locally definable subset of $W$ which is both $w$-open and $w$ closed and whose intersection with any definable subset of $W$ is definable. In view of Lemma 2.3, this notion generalizes the notion of definably connected in locally definable groups studied in [3].

Remark 2.8 Suppose that $w: W \longrightarrow G$ is a locally definable covering map and let $y \in W$ be such that $w(y)=e_{G}$. Suppose that $W$ and $G$ are definably $w$-connected and $\tau$-connected respectively. Then we have a 
well defined homomorphism $w_{*}: \pi_{1}(W) \longrightarrow \pi_{1}(G):[\gamma] \mapsto[w \circ \gamma]$ and the following hold.

(1) If $\sigma$ is a $\tau$-path in $G$ from $e_{G}$ to $e_{G}$, then $y=y * \sigma$ if and only if $[\sigma] \in w_{*}\left(\pi_{1}(W)\right)$.

(2) If $\sigma$ and $\sigma^{\prime}$ are two $\tau$-paths in $G$ from $e_{G}$ to $x$, then $y * \sigma=y * \sigma^{\prime}$ if and only if $\left[\sigma \cdot \sigma^{\prime-1}\right] \in w_{*}\left(\pi_{1}(W)\right)$.

Let $w: W \longrightarrow G$ be a locally definable covering map. We say that $W$ is $w$-path connected if for every $u, v \in W$ there is a $w$-path $\alpha:[0, q] \longrightarrow W$ such that $\alpha(0)=u$ and $\alpha(q)=v$.

Lemma 2.9 Let $w: W \longrightarrow G$ be a locally definable covering map. Then $W$ is definably $w$-connected if and only if $W$ is w-path connected. In fact, for any definably $w$-connected definable subset $X$ of $W$ there is a uniformly definable family of $w$-paths in $X$ connecting a given fixed point in $X$ to any other point in $X$.

Proof. Since $w: W \longrightarrow G$ is a locally definable covering map, it is enough to prove the result for locally definable groups. By the first part of the proof of [9] Lemma 2.13, there is a locally definable subset $U$ of $G$ such that $\operatorname{dim}(G \backslash U)<\operatorname{dim} G$, the intersection of any definable subset of $G$ with $U$ is a definable subset and the induced $\tau$-topology on $U$ coincides with the induced topology from $R^{n}$. So a definable subset $B$ of $U$ is $\tau$-connected if and only if $B$ is definably connected (in $R^{n}$ ). Thus the result follows from by [6] Chapter VI, Proposition 3.2 and its proof, saturation and [3] Lemma 3.5 (i.e., countably many translates of $U$ cover $G$ ).

The next proposition is also a consequence of Lemma 2.7 and is proved in exactly the same way as its definable analogue in [4] Corollary 2.8 and Proposition 2.10.

Proposition 2.10 Let $w: W \longrightarrow G$ be a locally definable covering map. Suppose that $W$ and $G$ are definably $w$-connected and $\tau$-connected respectively. Then the following hold:

(1) $w_{*}: \pi_{1}(W) \longrightarrow \pi_{1}(G)$ is an injective homomorphism;

(2) $\pi_{1}(G) / w_{*}\left(\pi_{1}(W)\right) \simeq \operatorname{Aut}(W / G)$ (the group of all locally definable $w$ homeomorphisms $\phi: W \longrightarrow W$ such that $w=w \circ \phi)$.

Below we will also require the following generalization of Lemma 2.6: 
Lemma 2.11 Let $w: W \longrightarrow G$ and $v: V \longrightarrow H$ be locally definable covering maps and let $f, g: V \longrightarrow W$ be two continuous locally definable maps (with respect to the $v$ and $w$ topologies) such that $w \circ f=w \circ g$. If $V$ is definably $v$-connected and $f(x)=g(x)$ for some $x \in V$, then $f=g$.

Proof. This is as in [3] Lemma 3.2 once we show that $\{x \in V: f(x)=$ $g(x)\}$, which is open and closed, is a locally definable subset whose intersection with any definable subset of $V$ is a definable subset of $V$. If $C, D \subseteq V$ are definable, then $\left(V \times{ }_{W} V\right) \cap(C \times D)=\left\{(x, y) \in C \times D: f_{\mid C}(x)=g_{\mid D}(y)\right\}$ is definable, and so $\left(V \times_{W} V\right) \cap E$ is definable for every definable subset $E$ of $V \times V$. Similarly, $\Delta_{V} \cap E$ is definable for every definable subset $E$ of $V \times V$. Hence, $\left(V \times_{W} V\right) \cap \Delta_{V} \cap E$ is definable for every definable subset $E$ of $V \times V$. From this and the observation (2) on page 5 we get our result since $\{x \in V: f(x)=g(x)\}=i^{-1}\left(\left(V \times_{W} V\right) \cap \Delta_{V}\right)$, where $i: V \longrightarrow \Delta_{V}: x \mapsto(x, x)$ is a locally definable map.

Finally we include the following result ([3] Proposition 3.4) which will also be useful later:

Proposition 2.12 Let $h: H \longrightarrow G$ be a locally definable covering homomorphism and suppose that $H$ is definably $\tau$-connected. Then

$$
\operatorname{Kerh} \simeq \operatorname{Aut}(H / G)
$$

and $\operatorname{Aut}(H / G)$ is abelian.

\section{The universal covering homomorphism}

Here we will present the proof of our main result. We start however with a special case.

\subsection{A special case of the main result}

The main result of the paper [5], in the language of the theory of locally definable covering homomorphisms, is the following (compare with [5] Remark 6.14). For a related result see also [8].

Theorem 3.1 ([5]) Suppose that $\mathcal{R}$ is an ordered vector space over an ordered division ring and $G$ is a definably t-connected, definably compact, definable group of dimension $n$. Then there is a locally definable group $V$ which is a subgroup of $\left(R^{n},+\right)$ and a locally definable covering homomorphism $v: V \longrightarrow G$ such that $\pi_{1}(G) \simeq \operatorname{Ker} v \simeq \mathbb{Z}^{n}$. 
In [5] Remark 6.14 it is suggested that $v: V \longrightarrow G$ is in some sense the universal cover of $G$ since we have $\pi_{1}(V)=1$ ([5] Corollary 6.7). This claim can now be made more precise:

Theorem 3.2 Suppose that $\mathcal{R}$ is an ordered vector space over an ordered division ring and $G$ is a definably t-connected, definably compact, definable group of dimension $n$. Then the locally definable covering homomorphism $v: V \longrightarrow G$ is isomorphic to $\widetilde{p}: \widetilde{G} \longrightarrow G$ and $\pi_{1}(G) \simeq \pi(G) \simeq \mathbb{Z}^{n}$.

Proof. Suppose that $q: K \longrightarrow V$ is a locally definable covering homomorphism. Then from Propositions 2.10 and 2.12 we obtain Ker $q \simeq$ $\operatorname{Aut}(K / V) \simeq \pi_{1}(V) / q_{*}\left(\pi_{1}(K)\right)=1$ since $\pi_{1}(V)=1$, by [5] Corollary 6.7. So $q: K \longrightarrow V$ is a locally definable isomorphism (since it is surjective). Consequently, by [3] Lemma 3.8, the set of all $h: H \longrightarrow G$ in $\operatorname{Cov}^{0}(G)$ which are locally definably isomorphic to $v: V \longrightarrow G$ is cofinal in $\operatorname{Cov}^{0}(G)$ and hence the inverse limit $\widetilde{p}: \widetilde{G} \longrightarrow G$ is isomorphic to $v: V \longrightarrow G$. By Propositions 2.10 and 2.12 we obtain $\pi(G) \simeq \operatorname{Ker} v \simeq \operatorname{Aut}(V / G) \simeq \pi_{1}(G)$ since $\pi_{1}(V)=1$. Thus the result holds as required.

\subsection{The main result}

Here we prove the main result of the paper. Before we proceed we need the following propositions.

Proposition 3.3 Let $G$ be a definably $\tau$-connected locally definable group of dimension $k$. Then there is a countable collection $\left\{O_{s}: s \in S\right\}$ of $\tau$-open definably $\tau$-connected definable subsets of $G$ with $G=\cup\left\{O_{s}: s \in S\right\}$ and, for each $s \in S, O_{s}$ is definably homeomorphic to an open cell in $R^{k}$. In particular, for each $s \in S$, the o-minimal fundamental group $\pi_{1}\left(O_{s}\right)$ with respect to the induced $\tau$-topology on $O_{s}$ is trivial

Proof. By the first part of the proof of [9] Lemma 2.13, there is a locally definable subset $U$ of $G$ such that $\operatorname{dim}(G \backslash U)<\operatorname{dim} G$, the intersection of any definable subset of $G$ with $U$ is a definable subset and the induced $\tau$-topology on $U$ coincides with the induced topology from $R^{n}$. Without loss of generality we can assume that $U$ is a countable union of cells of dimension $k=\operatorname{dim} G$. Note that on each of these $k$-cells in $U$, the induced $\tau$-topology coincides with the induced topology from $R^{n}$. By [3] Lemma 3.5 countably many translates of $U$ cover $G$, so countably many $\tau$-open definably $\tau$-connected subsets of $G$ which are definably $\tau$-homeomorphic to $k$-cells in $U$ cover $G$. 
Let $\left\{O_{s}: s \in S\right\}$ be this collection. To finish, it is enough to show that if $C$ is an open cell in $R^{k}$ then $\pi_{1}(C)=1$ (since definable homeomorphisms induce isomorphisms between the o-minimal fundamental groups).

We will show this by induction on the construction of cells. If $C$ has dimension zero then this is obvious. Assume that $C=(a, b) \subseteq R \cup\{-\infty,+\infty\}$ is an open cell of dimension one and $\alpha:[0, q] \longrightarrow C$ is a definable loop at $c \in C$. Consider the continuous definable map $H:[0, q] \times[0, q] \longrightarrow C$ given by

$$
H(t, x):=\alpha\left(\frac{t+x+|t-x|}{2}\right) .
$$

Then $H$ is a definable homotopy between $\alpha$ and $\epsilon_{c}$. So $[\alpha]=1$ and $\pi_{1}(C)=1$ as required.

Suppose that $B$ is a cell, $\pi_{1}(B)=1$ and $C=(f, g)_{B}$ with $f, g: B \longrightarrow R \cup$ $\{-\infty,+\infty\}$ continuous definable maps such that $f<g$. Let $c=(b, a) \in C$ and let $\sigma:[0, q] \longrightarrow C$ be a definable loop at $c$. We can write $\sigma(t)=$ $(\beta(t), \alpha(t))$ for some definable loop $\beta:[0, q] \longrightarrow B$ at $b$ and $\alpha:[0, q] \longrightarrow R$ a definable loop at $a$. By assumption there is a definable homotopy $F:[0, q] \times$ $[0, p] \longrightarrow B$ between $\beta$ and $\epsilon_{b}$ and a definable homotopy $E:[0, q] \times[0, r] \longrightarrow$ $R$ between $\alpha$ and $\epsilon_{a}$. Let $H:[0, q] \times[0, \max \{r, p\}] \longrightarrow C$ be the definable map such that if $r \leq p$ then

$$
H(t, x)= \begin{cases}(F(t, x), E(t, x)) & \text { if } x \leq r, \\ (F(t, x), E(t, r)) & \text { if } x \geq r\end{cases}
$$

and if $p \leq r$ then

$$
H(t, x)= \begin{cases}(F(t, x), E(t, x)) & \text { if } x \leq p, \\ (F(t, p), E(t, x)) & \text { if } x \geq p .\end{cases}
$$

Then $H$ is a definable homotopy between $\sigma$ and $\epsilon_{c}$. So $[\sigma]=1$ and $\pi_{1}(C)=1$ as required.

Proposition 3.4 Let $G$ be a definably $\tau$-connected locally definable group. Then the o-minimal fundamental group $\pi_{1}(G)$ of $G$ (with respect to the induced $\tau$-topology) is countable. In fact, if $G$ is definable, then $\pi_{1}(G)$ is finitely generated.

Proof. Consider the countable cover $\left\{O_{s}: s \in S\right\}$ of $G$ by $\tau$-open definably $\tau$-connected definable subsets given by Proposition 3.3. For each pair of distinct elements $s, t \in S$ such that $O_{s} \cap O_{t} \neq \emptyset$ and for each definably 
$\tau$-connected component $C$ of this intersection choose a point $a_{s, t, C} \in C$. For each pair $\left(a_{s, t, C}, a_{s^{\prime}, t^{\prime}, D}\right)$ of distinct points and $l \in\{s, t\} \cap\left\{s^{\prime}, t^{\prime}\right\}$ let $\sigma_{(C, D), s, t, s^{\prime}, t^{\prime}}^{l}$ be a $\tau$-path in $O_{l}$ from $a_{s, t, C}$ to $a_{s^{\prime}, t^{\prime}, D}$. Also, for each $a_{s, t, C}$ such that $e_{G} \in O_{s}$, let $\sigma_{\left(e_{G}, C\right), s, t}^{s}$ (respectively, $\left.\sigma_{\left(C, e_{G}\right), s, t}^{s}\right)$ be a $\tau$-path in $O_{s}$ from $e_{G}$ to $a_{s, t, C}$ (respectively, from $a_{s, t, C}$ to $e_{G}$ ).

Let $\Sigma$ be the countable collection of all $\tau$-paths $\sigma_{(C, D), s, t, s^{\prime}, t^{\prime}}^{l}, \sigma_{\left(e_{G}, C\right), s, t}^{s}$ and $\sigma_{\left(C, e_{G}\right), s, t}^{s}$ as above. The set $\Sigma$ generates a free countable language $\Sigma^{*}$ such that some of its words correspond in an obvious way to $\tau$-paths in $G$. To finish the proof it is enough to show that any $\tau$-loop in $G$ is $\tau$-homotopic to a $\tau$-loop which is a concatenation of $\tau$-paths in $\Sigma$ and thus corresponds to a word in $\Sigma^{*}$.

Let $\lambda$ be a $\tau$-loop in $G$. Then by saturation and o-minimality there exists a minimal $k$ for which we can choose points $0=t(0)<t(1)<\cdots<t(k)<$ $t(k+1)=q_{\lambda}$ such that for each $j=0, \ldots, k$, we have $\lambda([t(j), t(j+1)]) \subseteq O_{s(j)}$ for some $s(j) \in S$. Thus $\lambda=\lambda_{0} \cdots \cdots \lambda_{k}$ where, for each $j, \lambda_{j}:\left[0, q_{\lambda_{j}}\right] \longrightarrow G$ is the $\tau$-path with $q_{\lambda_{j}}=t(j+1)-t(j)$ and given by $\lambda_{j}(t)=\lambda(t+t(j))$. For $i=0, \ldots, k-1$, let $C_{i}$ be the definably $\tau$-connected component of $O_{s(i)} \cap$ $O_{s(i+1)}$ containing $\lambda_{i}\left(q_{\lambda_{i}}\right)$ and let $\epsilon_{i}$ be a $\tau$-path in $C_{i}$ from $a_{s(i), s(i+1), C_{i}}$ to $\lambda_{i}\left(q_{\lambda_{i}}\right)$. Let $\sigma_{0}$ be the $\tau$-path $\sigma_{\left(e_{G}, C_{0}\right), s(0), s(1)}^{s(n)} O_{s(0)}$ and let $\sigma_{k}$ be the $\tau$-path $\sigma_{\left(C_{k-1}, e_{G}\right), s(k-1), s(k)}^{s(k)}$ in $O_{s(k)}$. Finally, for $i=1, \ldots, k-1$, let $\sigma_{i}$ be the $\tau$-path $\sigma_{\left(C_{i-1}, C_{i}\right), s(i-1), s(i), s(i), s(i+1)}^{s(i)}$ in $O_{s(i)}$. Since by Proposition 3.3, $\pi_{1}\left(O_{s(j)}\right)=1$ for all $j=0, \ldots, k$, we have that $\sigma_{0}$ is $\tau$-homotopic to $\lambda_{0} \cdot \epsilon_{0}^{-1}, \sigma_{k}$ is $\tau$-homotopic to $\epsilon_{k-1} \cdot \lambda_{k}$ and, for each $i=1, \ldots, k-1, \sigma_{i}$ is $\tau$-homotopic to $\epsilon_{i-1} \cdot \lambda_{i} \cdot \epsilon_{i}^{-1}$. Hence, $\lambda$ is $\tau$-homotopic to $\sigma_{0} \cdot \sigma_{1} \cdots \cdots \sigma_{k} \in \Sigma^{*}$ as required.

Assume now that $G$ is definable. Let $K$ be the simplicial complex of dimension one whose vertices are the end points of the $\tau$-paths in $\Sigma$ and whose edges are the images of the $\tau$-paths in $\Sigma$. Clearly we have a homomorphism $\pi_{1}\left(|K|, e_{G}\right) \longrightarrow \pi_{1}(G)$ which sends an edge loop in $K$ into the $\tau$-loop it determines in $G$. This is well defined since if two edge loops are homotopic in $|K|$ then they are obviously $\tau$-homotopic in $G$. The argument in the previous paragraph shows that the homomorphism $\pi_{1}\left(|K|, e_{G}\right) \longrightarrow \pi_{1}(G)$ is surjective. Now as explained in [2] Chapter 3, Subsection 3.5.3, the fundamental group of a (finite) simplicial complex is finitely generated. Hence $\pi_{1}(G)$ is also finitely generated.

For the rest of the section, fix $G$ a definably $\tau$-connected locally definable group.

We will construct now an "abstract universal covering map" $u: U \longrightarrow G$ from which we will obtain a locally definable covering map $v: V \longrightarrow G$ which 
will be a locally definable covering homomorphism once we put a suitable locally definable group structure on $V$. The later will then be shown to be isomorphic to $\widetilde{p}: \widetilde{G} \longrightarrow G$.

Given two $\tau$-paths $\sigma:\left[0, q_{\sigma}\right] \longrightarrow G$ and $\lambda:\left[0, q_{\lambda}\right] \longrightarrow G$ in $G$, we put $\sigma \simeq \lambda$ if and only if $\sigma(0)=\lambda(0)=e_{G}, \sigma\left(q_{\sigma}\right)=\lambda\left(q_{\lambda}\right)$ and $\left[\sigma \cdot \lambda^{-1}\right]=1 \in$ $\pi_{1}(G)$. Here, $\lambda^{-1}:\left[0, q_{\lambda^{-1}}\right] \longrightarrow G$ is the $\tau$-path such that $q_{\lambda^{-1}}=q_{\lambda}$ and $\lambda^{-1}(t)=\lambda\left(q_{\lambda}-t\right)$ for every $t$ in $\left[0, q_{\lambda^{-1}}\right]$. The relation $\simeq$ is an equivalence relation and we denote the equivalence class of $\sigma$ under $\simeq$ by $\langle\sigma\rangle$. For each $s \in S$, let $U_{s}=\left\{\langle\sigma\rangle: \sigma\right.$ is a $\tau$-path in $G$ such that $\sigma(0)=e_{G}$ and $\left.\sigma\left(q_{\sigma}\right) \in O_{s}\right\}$ and fix a $\tau$-path $\sigma_{s}:\left[0, q_{s}\right] \longrightarrow G$ such that $\sigma(0)=e_{G}$ and $\sigma\left(q_{s}\right) \in O_{s}$.

Claim 3.5 There is a well-defined bijection

$$
\phi_{s}: U_{s} \longrightarrow O_{s} \times \pi_{1}(G):\langle\lambda\rangle \mapsto\left(\lambda\left(q_{\lambda}\right),\left[\lambda \cdot \eta \cdot \sigma_{s}^{-1}\right]\right),
$$

where $\eta:\left[0, q_{\eta}\right] \longrightarrow O_{s}$ is a $\tau$-path in $O_{s}$ such that $\eta(0)=\lambda\left(q_{\lambda}\right)$ and $\eta\left(q_{\eta}\right)=$ $\sigma_{s}\left(q_{s}\right)$.

Proof. Clearly, $\phi_{s}$ is well-defined, i.e. it does not depend on the choice of $\eta$ since $\pi_{1}\left(O_{s}\right)=1$ (Proposition 3.3) and for $\langle\lambda\rangle=\left\langle\lambda^{\prime}\right\rangle$ we have $\lambda\left(q_{\lambda}\right)=\lambda\left(q_{\lambda^{\prime}}\right)$ and

$$
\begin{aligned}
{\left[\lambda \cdot \eta \cdot \sigma_{s}^{-1}\right] } & =\left[\lambda \cdot \lambda^{\prime-1} \cdot \lambda^{\prime} \cdot \eta \cdot \sigma_{s}^{-1}\right] \\
& =\left[\lambda \cdot \lambda^{\prime-1}\right]\left[\lambda^{\prime} \cdot \eta \cdot \sigma_{s}^{-1}\right] \\
& =\left[\lambda^{\prime} \cdot \eta \cdot \sigma_{s}^{-1}\right] .
\end{aligned}
$$

Also, for $o \in O_{s}$ and $[\gamma] \in \pi_{1}(G)$ we have $\phi_{s}(\langle\lambda\rangle)=(o,[\gamma])$ for $\lambda=$ $\gamma \cdot \sigma_{s} \cdot \eta^{-1}$, where $\eta:\left[0, q_{\eta}\right] \longrightarrow G$ is a $\tau$-path in $O_{s}$ such that $\eta(0)=o$ and $\eta\left(q_{\eta}\right)=\sigma_{s}\left(q_{s}\right)$. Thus $\phi_{s}$ is surjective. On the other hand, suppose that $\phi_{s}(\langle\lambda\rangle)=\phi_{s}\left(\left\langle\lambda^{\prime}\right\rangle\right)$. Then $\lambda\left(q_{\lambda}\right)=\lambda^{\prime}\left(q_{\lambda^{\prime}}\right)$ and $\left[\lambda \cdot \eta \cdot \sigma_{s}^{-1}\right]=\left[\lambda^{\prime} \cdot \eta^{\prime} \cdot \sigma_{s}^{-1}\right]$. But we also have

$$
\begin{aligned}
{\left[\lambda \cdot \eta \cdot \sigma_{s}^{-1}\right] } & =\left[\lambda \cdot \lambda^{\prime-1}\right]\left[\lambda^{\prime} \cdot \eta \cdot \sigma_{s}^{-1}\right] \\
& =\left[\lambda \cdot \lambda^{\prime-1}\right]\left[\lambda^{\prime} \cdot \eta^{\prime} \cdot \sigma_{s}^{-1}\right] \\
& =\left[\lambda \cdot \lambda^{\prime-1}\right]\left[\lambda \cdot \eta \cdot \sigma_{s}^{-1}\right]
\end{aligned}
$$

(the fact $\pi_{1}\left(O_{s}\right)=1$ (Proposition 3.3) implies that $\lambda^{\prime} \cdot \eta \cdot \sigma_{s}^{-1}$ is $\tau$-homotopic to $\left.\lambda^{\prime} \cdot \eta^{\prime} \cdot \sigma_{s}^{-1}\right)$. Thus we have $\left[\lambda \cdot \lambda^{\prime-1}\right]=1,\langle\lambda\rangle=\left\langle\lambda^{\prime}\right\rangle$ and $\phi_{s}$ is injective.

Set $U=\cup\left\{U_{s}: s \in S\right\}$ and let $u: U \longrightarrow G$ be the surjective map given by $u(\langle\lambda\rangle)=\lambda\left(q_{\lambda}\right)$. By Claim 3.5 and its proof we have, for each $s \in S$,

$(\bullet) u^{-1}\left(O_{s}\right)$ is the disjoint union of the subsets $\phi_{s}^{-1}\left(O_{s} \times\{[\gamma]\}\right)$ with $[\gamma] \in$ $\pi_{1}(G)$; 
$(\bullet \bullet) u$ restricted to $\phi_{s}^{-1}\left(O_{s} \times\{[\gamma]\}\right)$ is a bijection onto $O_{s}$.

Claim 3.6 If $s, t \in S$ are such that $O_{s} \cap O_{t} \neq \emptyset$ and $C$ is a definably $\tau$ connected component of $O_{s} \cap O_{t}$, then the restriction of the bijection

$$
\phi_{t} \circ \phi_{s}^{-1}:\left(O_{s} \cap O_{t}\right) \times \pi_{1}(G) \longrightarrow\left(O_{s} \cap O_{t}\right) \times \pi_{1}(G)
$$

to $C \times\{[\gamma]\}$ is the same as $C \times\{[\gamma]\} \longrightarrow C \times\left\{\left[\gamma_{C}\right]\right\}:(o,[\gamma]) \mapsto\left(o,\left[\gamma_{C}\right]\right)$ for some $\left[\gamma_{C}\right] \in \pi_{1}(G)$.

Proof. Let $o \in C$. By Claim 3.5 and its proof , $\phi_{t} \circ \phi_{s}^{-1}(o,[\gamma])=\left(o,\left[\lambda \cdot \eta^{\prime}\right.\right.$. $\left.\left.\sigma_{t}^{-1}\right]\right)$, where $\lambda=\gamma \cdot \sigma_{s} \cdot \eta^{-1}$ and $\eta:\left[0, q_{\eta}\right] \longrightarrow O_{s}$ and $\eta^{\prime}:\left[0, q_{\eta^{\prime}}\right] \longrightarrow O_{t}$ are $\tau$ paths such that $\eta(0)=\eta^{\prime}(0)=o, \eta\left(q_{\eta}\right)=\sigma_{s}\left(q_{s}\right)$ and $\eta^{\prime}\left(q_{\eta^{\prime}}\right)=\sigma_{t}\left(q_{t}\right)$. Thus to prove the claim it is enough to show that $\left[\gamma \cdot \sigma_{s} \cdot \eta^{-1} \cdot \eta^{\prime} \cdot \sigma_{t}^{-1}\right]=\left[\gamma \cdot \sigma_{s} \cdot \theta^{-1} \cdot \theta^{\prime} \cdot \sigma_{t}^{-1}\right]$ whenever $\theta:\left[0, q_{\theta}\right] \longrightarrow O_{s}$ and $\theta^{\prime}:\left[0, q_{\theta^{\prime}}\right] \longrightarrow O_{t}$ are $\tau$-paths such that $\theta(0)=\theta^{\prime}(0) \in C, \theta\left(q_{\theta}\right)=\sigma_{s}\left(q_{s}\right)$ and $\theta^{\prime}\left(q_{\theta^{\prime}}\right)=\sigma_{t}\left(q_{t}\right)$.

Since $C$ is $\tau$-path connected, let $\rho:\left[0, q_{\rho}\right] \longrightarrow C$ be a $\tau$-path such that $\rho(0)=o$ and $\rho\left(q_{\rho}\right)=\theta(0)=\theta^{\prime}(0)$. Now using the fact that $\pi_{1}\left(O_{s}\right)=$ $\pi_{1}\left(O_{t}\right)=1$ (Proposition 3.3) we see that $\rho \cdot \theta$ (respectively $\theta^{\prime} \cdot \rho^{-1}$ ) is $\tau$ homotopic to $\eta$ (respectively $\eta^{-1}$ ). Thus $\eta^{-1} \cdot \eta^{\prime}$ is $\tau$-homotopic to $\theta^{-1} \cdot \theta^{\prime}$. From here we get $\left[\gamma \cdot \sigma_{s} \cdot \eta^{-1} \cdot \eta^{\prime} \cdot \sigma_{t}^{-1}\right]=\left[\gamma \cdot \sigma_{s} \cdot \theta^{-1} \cdot \theta^{\prime} \cdot \sigma_{t}^{-1}\right]$ as required.

We will let $1 \in R$ be a fixed 0 -definable positive element of $R$ and denote the element $n \cdot 1$ of the group $(R, 0,+)$ by $n$. By Proposition 3.4, we will identify $\pi_{1}(G)$ with a subset of $\mathbb{N} \subseteq R$ and thus, assuming that $G \subseteq R^{l}$,

$$
O_{(s,[\gamma])}:=O_{s} \times\{[\gamma]\}
$$

is a definable subset of $R^{l+1}$ and $O:=\cup\left\{O_{(s,[\gamma])}:(s,[\gamma]) \in S \times \pi_{1}(G)\right\}$ is a locally definable subset of $R^{l+1}$.

Let $\left\{\left(s_{i}, l_{j}\right):(i, j) \in \mathbb{N} \times \mathbb{N}\right\}$ be an enumeration of $S \times \pi_{1}(G)$. Define inductively (on $i$ ) the sets $N_{i}, O_{\left(s_{i}, l_{j}\right)}^{\prime}$ and $V_{\left(s_{i}, l_{j}\right)}$ in the following way:

$$
N_{0}=\emptyset \text { and } O_{\left(s_{0}, l_{j}\right)}^{\prime}=V_{\left(s_{0}, l_{j}\right)}=O_{\left(s_{0}, l_{j}\right)} ;
$$

assuming that $N_{i}, O_{\left(s_{i}, l_{j}\right)}^{\prime}$ and $V_{\left(s_{i}, l_{j}\right)}$ were already defined, put

$$
N_{i+1}=\left\{n: n<i+1 \text { and } O_{s_{i+1}} \cap O_{s_{n}} \neq \emptyset\right\}
$$

$O_{\left(s_{i+1}, l_{j}\right)}^{\prime}=O_{\left(s_{i+1}, l_{j}\right)} \backslash \cup\left\{C \times\left\{l_{j}\right\}: C\right.$ is a definably $\tau$-connected component of $O_{s_{i+1}} \cap O_{s_{n}}, n \in N_{i+1}$ and $\left.\left(\phi_{s_{i+1}} \circ \phi_{s_{n}}^{-1}\right)_{\mid C \times\left\{l_{C}\right\}}\left(o, l_{C}\right)=\left(o, l_{j}\right)\right\}$;

$V_{\left(s_{i+1}, l_{j}\right)}=O_{\left(s_{i+1}, l_{j}\right)}^{\prime} \cup \bigcup\left\{V_{\left(s_{n}, l_{C}\right)}^{C}: C\right.$ is a definably $\tau$-connected component of $O_{s_{i+1}} \cap O_{s_{n}}, n \in N_{i+1}$ and $\left.\left(\phi_{s_{i+1}} \circ \phi_{s_{n}}^{-1}\right)_{\mid C \times\left\{l_{C}\right\}}\left(o, l_{C}\right)=\left(o, l_{j}\right)\right\}$, where $V_{\left(s_{n}, l_{C}\right)}^{C}=\left\{x \in V_{\left(s_{n}, l_{C}\right)}: x=(o, l)\right.$ with $\left.o \in C\right\}$.

\footnotetext{
${ }^{1}$ We wish to thank here Elias Baro (Universidad Autónoma de Madrid) for pointing out an imprecision on an early version of our inductive construction.
} 
By Claim 3.6, the sets $V_{\left(s_{i}, l_{j}\right)}$ are well defined definable subsets of $R^{l+1}$.

Claim 3.7 Let $V=\cup\left\{V_{\left(s_{i}, l_{j}\right)}:(i, j) \in \mathbb{N} \times \mathbb{N}\right\}$. Then $V$ is a locally definable set and the surjective map $v: V \longrightarrow G$ given by the projection onto the first coordinate is a locally definable covering map, i.e., for each $i$, we have:

(1) $v^{-1}\left(O_{s_{i}}\right)=\cup\left\{V_{\left(s_{i}, l_{j}\right)}: j \in \mathbb{N}\right\}$ (disjoint union);

(2) $v_{\mid V_{\left(s_{i}, l_{j}\right)}}$ is a definable bijection onto $O_{s_{i}}$.

Proof. This follows by induction on the definition of the definable sets $V_{\left(s_{i}, l_{j}\right)}$ together with Claim 3.6.

Fix $s_{e_{G}} \in S$ such that $e_{G} \in O_{s_{e_{G}}}$ and assume without loss of generality that $\sigma_{s_{e_{G}}}=\epsilon_{e_{G}}\left(\right.$ the trivial $\tau$-loop at $e_{G}$, see page 7). Let $e_{V}=\left(e_{G},\left[\epsilon_{e_{G}}\right]\right) \in V$.

Claim 3.8 Let $(o,[\gamma]) \in V$ and suppose that $\lambda:\left[0, q_{\lambda}\right] \longrightarrow G$ is a $\tau$-path such that $\lambda(0)=e_{G}, \lambda\left(q_{\lambda}\right)=o$ and $\phi_{s}(\langle\lambda\rangle)=(o,[\gamma])$. Then there exists a $v$ path $\tilde{\lambda}:\left[0, q_{\tilde{\lambda}}\right] \longrightarrow V$ in $V$ such that $\widetilde{\lambda}(0)=e_{V}, \widetilde{\lambda}\left(q_{\tilde{\lambda}}\right)=(o,[\gamma])$ and $v \circ \widetilde{\lambda}=\lambda$. In particular, $V$ is $v$-path connected and the o-minimal fundamental group $\pi_{1}(V)$ of $V$ with respect to the $v$-topology is trivial.

Proof. By saturation and o-minimality there exists a minimal $k$ for which we can choose points $0=t(0)<t(1)<\cdots<t(k)<t(k+1)=q_{\lambda}$ such that for each $j=0, \ldots, k$, we have $\lambda([t(j), t(j+1)]) \subseteq O_{s(j)}$ for some $s(j) \in S$.

We prove the result by induction on $k$. If $k=0$, then $\lambda\left(\left[0, q_{\lambda}\right]\right) \subseteq O_{s(0)}$ and $[\gamma]=\left[\epsilon_{e_{G}}\right]$, and we put $\widetilde{\lambda}:=\left(v_{\mid V_{\left(s(0),\left[\epsilon_{G}\right]\right)}}\right)^{-1} \circ \lambda$. For the inductive step let $\eta:=\lambda_{\mid[0, t(k)]}$ and $\delta:\left[0, q_{\lambda}-t(k)\right] \longrightarrow O_{s(k)}: t \mapsto \lambda(t+t(k))$. By the induction hypothesis, let $\widetilde{\eta}:[0, t(k)] \longrightarrow V$ be a $v$-path such that $\widetilde{\eta}(0)=e_{V}$, $\widetilde{\eta}(t(k))=\left(\eta(t(k)),\left[\gamma^{\prime}\right]\right)$ and $v \circ \widetilde{\eta}=\eta$, where $\phi_{s(k-1)}(\langle\eta\rangle)=\left(\eta(t(k)),\left[\gamma^{\prime}\right]\right)$. Assume that $s(k)$ appear after $s(k-1)$ in the enumeration of $S$ introduced before. The other case is treated symmetrically. If $\phi_{s(k)}(\langle\eta\rangle)=\left(\eta(t(k)),\left[\gamma^{\prime \prime}\right]\right)$, then $\left(\eta(t(k)),\left[\gamma^{\prime}\right]\right)$ and $\left(\eta(t(k)),\left[\gamma^{\prime \prime}\right]\right)$ are the same point in $V_{\left(s(k),\left[\gamma^{\prime \prime}\right]\right)}$. Since $\lambda=\eta \cdot \delta$ and $\pi_{1}\left(O_{s(k)}\right)=1$ (Proposition 3.3), we have $[\gamma]=\left[\gamma^{\prime \prime}\right]$. Thus, if $\widetilde{\delta}:=\left(v_{\mid V_{\left(s(k),\left[\gamma^{\prime \prime}\right]\right)}}\right)^{-1} \circ \delta$, then $\widetilde{\eta}(t(k))=\widetilde{\delta}(0)$, and $\widetilde{\lambda}:=\widetilde{\eta} \cdot \widetilde{\delta}$ satisfies the claim. So, in particular, $V$ is $v$-path connected.

By Lemma 2.7, any $v$-loop $\delta$ in $V$ at $e_{V}$ is the unique lifting $\tilde{\lambda}$ of a $\tau$ loop $\lambda=v \circ \delta$ in $G$ at $e_{G}$ as defined in the previous paragraph. So we see that $\left(e_{G},\left[\epsilon_{e_{G}}\right]\right)=e_{V}=\widetilde{\lambda}(0)$ and $e_{V}=\widetilde{\lambda}\left(q_{\tilde{\lambda}}\right)=\left(e_{G},[\lambda]\right)$. This implies that $[\lambda]=1$ and so $v_{*}([\widetilde{\lambda}])=[\lambda]=1$. Therefore, since by Proposition 2.10 (i), $v_{*}: \pi_{1}(V) \longrightarrow \pi_{1}(G)$ is injective, it follows that $\pi_{1}(V)=1$. 
Our next goal is to make the locally definable covering map $v: V \longrightarrow G$ into a locally definable covering homomorphism. For this we will need the following claim:

Claim 3.9 Let $h: Y \longrightarrow X$ be either $v: V \longrightarrow G$ or $(v, v): V \times V \longrightarrow$ $G \times G$, and let $e_{Y}$ be $e_{V}$ or $\left(e_{V}, e_{V}\right)$ respectively, and $e_{X}$ be $e_{G}$ or $\left(e_{G}, e_{G}\right)$ respectively. Suppose that $g: X \longrightarrow G$ is a continuous locally definable map such that $g\left(e_{X}\right)=e_{G}$. Then there is a unique continuous locally definable map $\widetilde{g}: Y \longrightarrow V$ such that $\widetilde{g}\left(e_{Y}\right)=e_{V}$ and $v \circ \widetilde{g}=g \circ h$.

Proof. The uniqueness of such a locally definable lifting $\widetilde{g}$ of $g \circ h$ follows from Lemma 2.11. To construct $\widetilde{g}: Y \longrightarrow V$ we will use the fact that $h: Y \longrightarrow X$ is a locally definable covering map, and by Lemma 2.5 and Claim 3.8, $\pi_{1}(V \times V) \simeq \pi_{1}(V) \times \pi_{1}(V)=1$. We will also use the notation introduced right after Lemma 2.7.

Let $\left\{U_{l}: l \in L\right\}$ be either $\left\{O_{s}: s \in S\right\}$ or $\left\{O_{s} \times O_{t}: s, t \in S\right\}$. Let $f=g \circ h: Y \longrightarrow G$ and for each $l \in L$, let $\left\{V_{i}^{l}: i \in I_{l}\right\}$ be the definably $h$-connected components of $f^{-1}\left(U_{l}\right)$. For all $l \in L, i \in I_{l}$, choose $y_{i}^{l} \in V_{i}^{l}$ such that if $e_{Y} \in V_{i}^{l}$ then $e_{Y}=y_{i}^{l}$, and let $\eta_{i}^{l}$ be an $h$-path in $Y$ from $e_{Y}$ to $y_{i}^{l}$. Since each $V_{i}^{l}$ is definably $h$-connected, by Lemma 2.9 there is a uniformly definable family $\left\{\gamma_{i}^{l}(w): w \in V_{i}^{l}\right\}$ of $h$-paths in $V_{i}^{l}$ from $y_{i}^{l}$ to $w$. For $w \in V_{i}^{l}$, let $\delta_{i}^{l}(w)$ be the $h$-path $\eta_{i}^{l} \cdot \gamma_{i}^{l}(w)$ from $e_{Y}$ to $w$. Let $\sigma_{i}^{l}(w)=f \circ \delta_{i}^{l}(w)$ and put $\widetilde{f}(w)=e_{Y} * \sigma_{i}^{l}(w)$.

If $w \in V_{i}^{l} \cap V_{j}^{k}$ then we have another $h$-path $\delta_{j}^{k}(w)$ from $e_{Y}$ to $w$ obtained from $V_{j}^{k}$, and $f \circ\left(\delta_{j}^{k}(w) \cdot\left(\delta_{i}^{l}(w)\right)^{-1}\right)=\sigma_{j}^{k}(w) \cdot\left(\sigma_{i}^{l}(w)\right)^{-1}$ is a $\tau$-path from $e_{G}$ to $e_{G}$. By hypothesis, $\left[\sigma_{j}^{k}(w) \cdot\left(\sigma_{i}^{l}(w)\right)^{-1}\right] \in f_{*}\left(\pi_{1}(Y)\right)=1$ and by Remark $2.8(2), e_{Y} * \sigma_{i}^{l}(w)=e_{Y} * \sigma_{j}^{k}(w)$ and so $\tilde{f}$ is well defined. Note that the same argument shows that $\widetilde{f}$ does not depend on the choice of the points $y_{i}^{l} \in V_{i}^{l}$ or of the $h$-paths $\eta_{i}^{l}$.

We now show that $\tilde{f}$ is a locally definable map. For this it is enough to show that $\widetilde{f}_{\mid V_{i}^{l}}$ is a definable map since by saturation any definable subset of $Y$ is contained in a finite union of $V_{i}^{l}$ 's. But for $w \in V_{i}^{l}$, we have $\widetilde{f}(w)=$ $e_{Y} * \sigma_{i}^{l}(w)$ which is the endpoint of the lifting $\widetilde{\sigma_{j}^{l}(w)}$ of $\sigma_{j}^{l}(w)$ starting at $e_{Y}$. Since $\sigma_{j}^{l}(w)=\left(f \circ \eta_{i}^{l}\right) \cdot\left(f \circ \gamma_{i}^{l}(w)\right), \tilde{f}(w)$ is the endpoint of the lifting $\widetilde{f \circ \gamma_{i}^{l}(w)}$ of $f \circ \gamma_{i}^{l}(w)$ starting at the endpoint $\widetilde{f \circ \eta_{i}^{l}}\left(q_{\eta_{i}^{l}}\right)$ of the lifting $\widetilde{f \circ \eta_{i}^{l}}$ of $f \circ \eta_{i}^{l}$. Thus, if $W_{i}^{l}$ is a $v$-open subset of $v^{-1}\left(O_{l}\right)$ such that $v_{\mid W_{i}^{l}}: W_{i}^{l} \longrightarrow O_{l}$ is a definable homeomorphism and $\widetilde{f \circ \eta_{i}^{l}}\left(q_{\eta_{i}^{l}}\right) \in W_{i}^{l}$, then $\tilde{f}(w)=\left(\left(v_{\mid W_{i}^{l}}\right)^{-1} \circ(f \circ\right.$ $\left.\left.\gamma_{i}^{l}(w)\right)\right)\left(q_{\gamma_{i}^{l}(w)}\right)$ where $q_{\gamma_{i}^{l}(w)}$ is the end point of the domain of $\gamma_{i}^{l}(w)^{l}$. 
To finish we need to show that $\widetilde{g}:=\widetilde{f}$ is continuous. For this we use $v \circ \widetilde{g}=g \circ h=f$ (which is immediate from the above characterization of

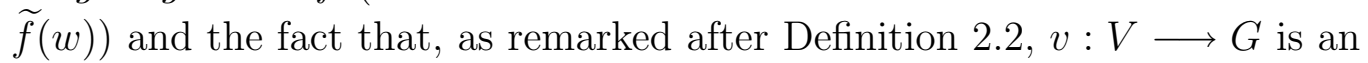
open mapping.

Let $\mu: G \times G \longrightarrow G$ and $\iota: G \longrightarrow G$ be the multiplication and the inverse in $G$. Let $\widetilde{\mu}: V \times V \longrightarrow V$ and $\widetilde{\iota}: V \longrightarrow V$ be the unique continuous locally definable maps given by Claim 3.9.

Claim $3.10\left(V, \widetilde{\mu}, \widetilde{\iota}, e_{V}\right)$ is a locally definable group and $v: V \longrightarrow G$ is a locally definable covering homomorphism.

Proof. We have that $\widetilde{\mu} \circ\left(\widetilde{\mu} \times \mathrm{id}_{V}\right)$ and $\widetilde{\mu} \circ\left(\mathrm{id}_{V} \times \widetilde{\mu}\right)$ are the liftings of the same continuous locally definable map $\mu \circ\left(\mu \times \operatorname{id}_{G}\right)=\mu \circ\left(\operatorname{id}_{G} \times \mu\right)$ and they coincide at $\left(e_{V}, e_{V}, e_{V}\right)$. Thus by Lemma 2.11 , we have $\widetilde{\mu} \circ\left(\widetilde{\mu} \times \mathrm{id}_{V}\right)=$ $\widetilde{\mu} \circ\left(\operatorname{id}_{V} \times \widetilde{\mu}\right)$ and so $(V, \widetilde{\mu})$ is a locally definable semigroup. Similarly, we see that $\tilde{\mu} \circ\left(\widetilde{\iota} \times \operatorname{id}_{V}\right) \circ \Delta_{V}=e_{V}=\widetilde{\mu} \circ\left(\operatorname{id}_{V} \times \widetilde{\iota}\right) \circ \Delta_{V}$ and $\widetilde{\mu} \circ i_{1}^{V}=\operatorname{id}_{V}=\widetilde{\mu} \circ i_{2}^{V}$ where $\Delta_{V}: V \longrightarrow V \times V$ is the diagonal map, $i_{1}^{V}: V \longrightarrow V \times V: v \mapsto\left(v, e_{V}\right)$ and $i_{2}^{V}: V \longrightarrow V \times V: v \mapsto\left(e_{V}, v\right)$. Thus $\left(V, \widetilde{\mu}, \widetilde{\iota}, e_{V}\right)$ is a locally definable group as required. Since $v \circ \widetilde{\mu}=\mu \circ(v, v)$ and $v \circ \widetilde{\iota}=\iota \circ v$, it follows that $v: V \longrightarrow G$ is a locally definable homomorphism which must be a locally definable covering homomorphism since it is also a locally definable covering map.

We are now ready to prove the main theorem of the paper (Theorem 1.4 in the introduction is a special case of this):

Theorem 3.11 Let $G$ be a definably $\tau$-connected locally definable group. Then the o-minimal universal covering homomorphism $\widetilde{p}: \widetilde{G} \longrightarrow G$ is a locally definable covering homomorphism and $\pi_{1}(G)$ is isomorphic to $\pi(G)$.

Proof. Suppose that $q: K \longrightarrow V$ is a locally definable covering homomorphism. Then from Propositions 2.10 and 2.12 we obtain Ker $q \simeq$ $\operatorname{Aut}(K / V) \simeq \pi_{1}(V) / q_{*}\left(\pi_{1}(K)\right)=1$ since $\pi_{1}(V)=1$, by Claim 3.8. So $q: K \longrightarrow V$ is a locally definable isomorphism (since it is surjective). Consequently, by [3] Lemma 3.8, the set of all $h: H \longrightarrow G$ in $\operatorname{Cov}^{0}(G)$ which are locally definably isomorphic to $v: V \longrightarrow G$ is cofinal in $\operatorname{Cov}^{0}(G)$ and hence the inverse limit $\widetilde{p}: \widetilde{G} \longrightarrow G$ is isomorphic to $v: V \longrightarrow G$. By Propositions 2.10 and 2.12 we obtain $\pi(G) \simeq \operatorname{Ker} v \simeq \operatorname{Aut}(V / G) \simeq \pi_{1}(G)$ since $\pi_{1}(V)=1$. Thus the result holds as required. 
Proof of Corollary 1.5: Let $G$ be a definably $t$-connected definable group. By Proposition 3.4, $\pi_{1}(G)$ is finitely generated and, by the isomorphism $\pi_{1}(G) \simeq \pi(G)$ (Theorem 3.11) and [3] Proposition 3.11, $\pi_{1}(G)$ is abelian. If $G$ is abelian, then by [12] the assumptions of [3] Theorem 3.15 hold for $G$. Therefore we have $\pi_{1}(G) \simeq \pi(G) \simeq \mathbb{Z}^{l}$ and $G[k] \simeq(\mathbb{Z} / k \mathbb{Z})^{l}$ for some $l \in \mathbb{N}$ as required.

\section{References}

[1] A. Berarducci and M. Otero O-minimal fundamental group, homology and manifolds J. London Math. Soc. 65 (2) (2002) 257-270.

[2] C. T. J. Dodson and P. E. Parker A User's Guide to Algebraic Topology Kluwer Academic Press 1997.

[3] M. Edmundo Covers of groups definable in o-minimal structures Illinois J. Math. 49 (1) (2005) 99-120.

[4] M. Edmundo and M. Otero Definably compact abelian groups J. Math. Logic 4 (2) (2004) 163-180.

[5] P. Eleftheriou and S. Starchenko Groups definable in ordered vector spaces over ordered division rings J. Symb. Logic (to appear).

[6] L. van den Dries Tame Topology and o-minimal Structures Cambridge University Press 1998.

[7] A. Hatcher Algebraic Topology Cambridge University Press 2002.

[8] A. Onshuus Groups definable in $(\mathbb{Z},+,<)$ Preprint 2005.

[9] Y. Peterzil and S. Starchenko Definable homomorphisms of abelian groups definable in o-minimal structures Ann. Pure Appl. Logic 101 (1) (1999) 1-27.

[10] A. Pillay On groups and fields definable in o-minimal structures J. Pure Appl. Algebra 53 (1988) 239-255.

[11] L. Ribes and P. Zalesskii Profinite Groups A Series of Modern Surveys in Mathematics, Volume 40, Springer 2000. 
[12] A.Strzebonski Euler characteristic in semialgebraic and other o-minimal groups J. Pure Appl. Algebra 96 (1994) 173-201. 